Ciência Florestal, Santa Maria, v. 21, n. 2, p. 349-354, abr.-jun., 2011

ISSN 0103-9954

\title{
AVALIAÇÃO DE SAUVEIROS EXTERNOS EM EUCALIPTAIS DE MINAS GERAIS
}

\section{LEAF CUTTING ANTS OUTSIDE THE EUCALYPT PLANTATIONS}

Iris Cristiane Magistrali ${ }^{1}$ Norivaldo dos Anjos $^{2}$

\section{RESUMO}

Existe uma grande dificuldade em aperfeiçoar as técnicas de manejo de formigas cortadeiras, sobretudo no que diz respeito aos sauveiros externos localizados próximos a plantios florestais. Por causa disso, o presente trabalho teve por objetivo caracterizar aspectos como tamanho e distância de sauveiros externos em plantações de eucaliptos. A avaliação foi realizada em aceiros externos de três fazendas localizadas nos municípios de João Pinheiro e Buritizeiro, em Minas Gerais, seis meses após ser realizado o combate convencional às formigas cortadeiras. Foram encontrados nove sauveiros externos, com área média de terra solta de $62,5 \pm 11,1 \mathrm{~m}^{2}$, localizados a uma distância média de 33,94 $\pm 8,68$ metros entre a margem do plantio e o local do formigueiro. Constatou-se que tais características podem ser úteis no ajuste da largura da faixa de segurança de combate, a qual deve ser igual a $50 \mathrm{~m}$. O gênero Trachymyrmex e as espécies Atta laevigata e Atta sexdens rubropilosa foram registrados como ocorrências novas no município de Buritizeiro, e o gênero Acromyrmex como uma nova ocorrência nos dois municípios.

Palavras-chave: pragas florestais; saúva; eucalipto.

\begin{abstract}
There is great difficulty to improve techniques of integrated pest management of leaf cutting ants, mainly when found outside of, but near to, forest plantations. This work aims to characterize aspects such as nest size and foraging distance from eucalypt plantations. Studies were performed near forest plantations in the counties of João Pinheiro and Buritizeiro, in Minas Gerais State, Brazil. Six months before, all leaf cutting ant nests received chemical control with sulfluramid baits. Nine nests were located outside the eucalypt plantations. Nest area was $62.5 \pm 11.1 \mathrm{~m}^{2}$ and nests were located $33.94 \pm 8.68 \mathrm{~m}$ from plantation boundary. Such characteristics may be useful to fix the boundary range used to control leaf cutting ant mounds outside the eucalypt plantation. Results show that boundary range should be $50 \mathrm{~m}$ wide. Acromyrmex sp. is a new occurrence for both Buritizeiro and João Pinheiro counties, but Atta laevigata, Atta sexdens rubropilosa and Trachymyrmex sp. are new occurrences for Buritizeiro only.
\end{abstract}

Keywords: forest pest; plantation boundary; eucalypt.

\section{INTRODUÇ̃̃O}

As formigas cortadeiras, com destaque para os gêneros Atta (saúva) e Acromyrmex (quenquém), atacam e desfolham árvores de várias espécies florestais provocando perdas consideráveis na produção, o que as destacam como sendo a principal praga florestal no Brasil. Árvores de Eucalyptus grandis, por exemplo, se completamente desfolhadas, aos 6 meses de idade, podem apresentar perdas de até $13 \%$ na produção volumétrica de madeira, ao final de sete anos (OLIVEIRA, 1996).

Entre as saúvas, a espécie Atta sexdens rubropilosa Forel, 1908 é a mais importante, sob o ponto de vista econômico, porque é muito agressiva às árvores e ocorre na maior parte das culturas florestais brasileiras. Trata-se da espécie de ocorrência mais ampla no País e pode ser

1. Engenheira Florestal. Mestranda pelo Programa de Pós-Graduação em Engenharia Florestal, Centro de Ciências Rurais, Universidade Federal de Santa Maria, Av Roraima, 1000, CEP 97105-970, Santa Maria (RS). irismagistrali@gmail.com

2. Engenheiro Florestal, Dr., Professor Titular em Manejo de Pragas Florestais, Casa dos Cupins - DBA/CCB, Universidade Federal de Viçosa, Av. P. H. Rolfs s/n, CEP 36570-000, Viçosa (MG). nanjos@ufv.br

Recebido para publicação em 12/03/2009 e aceito em 12/08/2010.

Ci. Fl., v. 21, n. 2, abr.-jun., 2011 
considerada como a mais comum, estando ausente somente na caatinga, em grande parte do Paraná, no litoral de Santa Catarina e em parte do Nordeste e do Rio Grande do Sul (DELLA LUCIA et al., 2000). Segundo Anjos et al. (1998), encontra-se distribuída nos estados de Minas Gerais, Espírito Santo, Rio de Janeiro, São Paulo, Goiás, Mato Grosso, Paraná e, de acordo com Oliveira (1996), também na Bahia. Essa espécie de saúva já foi registrada atacando árvores de Hevea brasiliensis Muell. Arg., Cabralea canjerana (Vell) Mart, Acacia mangium Willd, Azadirachta indica A. Juss, Eucalyptus grandis Hill ex Maiden, Eucalyptus urophylla S. T. Blake, Eucalyptus camaldulensis Dehn, Eucalyptus saligna Smith e Corymbia citriodora (Hook), conforme relataram Silva (2001), Garcia e Forti (1995), Bergmann et al. (1995), Garcia et al. (2001), Marsaro Junior (2005) e Moreira (2006).

A segunda espécie de saúva de importância econômica no Brasil é Atta laevigata (F. Smith, 1858), conforme afirmaram Anjos et al. (1998). Essa espécie já foi encontrada atacando espécies florestais como Atta mangium, Annona coriacea Mart, Casearia sp., Cecropia purpurascens Berg, Rollinia exsucca (DC. Ex Dunnal), Hancornia speciosa Gomez e Kielmeyera coriacea Mart. ex Saddi., Eucalyptus camaldulensis entre muitas outras, conforme trabalhos de Schoereder (1985), Vasconcelos (1997), Marsaro Junior (2005) e Grandeza et al. (1999). De acordo com Della Lucia et al. (2000), sua ocorrência já foi registrada no Amazonas, Paraíba, Pará, Tocantins, Ceará, Maranhão, Pernambuco, Alagoas, Sergipe, Bahia, Minas Gerais, Rio de Janeiro, São Paulo, Goiás, Mato Grosso, Mato Grosso do Sul e Rondônia.

Para evitar a inviabilização da cultura dos eucaliptos, é imprescindível que se faça o combate dos sauveiros nas áreas plantadas e no seu entorno. Os sauveiros que atacam o povoamento florestal, mas ficam localizados na vizinhança, em especial em remanescentes de vegetação nativa, em pastagens, plantações abandonadas, culturas agrícolas e outros ambientes, são aqui denominados de "sauveiros externos". Lima e Anjos (1997) haviam constatado que em $37,31 \%$ dos casos de monitoramentos de formigas cortadeiras, ocorreram ataques de sauveiros externos aos eucaliptais e o tipo de vizinhança com maior frequência de sauveiros externos foi o de mata natural, representando $84 \%$ dos casos. Ainda segundo esses autores, 88\% desses sauveiros externos localizavam-se dentro da vegetação natural, a uma distância de até 50 metros da margem do plantio de eucaliptos, sendo que, em até 10 metros de distância, se localizavam a metade dos casos constatados.

Um sauveiro externo pode ser caracterizado por apresentar, geralmente, um enorme monte de terra solta com grande quantidade de olheiros ativos, situados em pontos de acesso muito difícil, mas facilmente detectável através das várias trilhas de forrageamento que o conecta com as árvores atacadas pelas formigas. Tais sauveiros podem estar localizados em distâncias de até 400 metros das árvores atacadas (MARICONI et al., 1970). Lima e Anjos (1997) constataram que todos os formigueiros externos encontrados nas operações de monitoramento em eucaliptais forrageavam a uma distância de no máximo 100 metros. De acordo com esses autores, o combate nas áreas de vizinhança pode ser considerado como seguro para a grande maioria dos sauveiros externos, se realizado numa faixa com largura de até 50 metros de distância dos eucaliptais. Não existem trabalhos que indiquem qual é a largura ideal da faixa de terreno necessário para ser ter segurança contra o ataque de formigas cortadeiras provenientes da vizinhança. Por outro lado, não existe nenhuma avaliação sobre a funcionalidade da informação gerada por Lima e Anjos (1997) nos diversos plantios florestais em que tem sido adotada essa recomendação.

Neste trabalho, procurou-se caracterizar os sauveiros externos remanescentes às plantações de eucaliptos, após fazer o combate geral de formigas cortadeiras.

\section{MATERIAL E MÉTODOS}

Os dados foram obtidos em plantações comerciais de eucaliptos que estavam ladeados por vizinhança constituída de capinzais, arbustos e árvores de portes variados que formavam vegetações nativas típicas do cerrado, como os campos limpos, os campos sujos, os cerradinhos e os cerradões de grande porte. No mês de fevereiro de 2008, examinaram-se 21 talhões em 7.768 ha de efetivo plantio com as espécies de Eucalyptus urophylla e de Eucalyptus camaldulensis, em diferentes idades. Os plantios estavam localizados em três fazendas, das quais a primeira tinha 3.700 ha e estava no município de João Pinheiro; a segunda e terceira tinham 1.849 ha e 2.219 ha respectivamente, e estavam localizadas no município de Buritizeiro, Minas Gerais. Nessas plantações, o combate foi realizado nos meses de julho e agosto de 2007, por

Ci. Fl., v. 21, n. 2, abr.-jun., 2011 
meio do sistema "Arrastão", em que os operários colocaram de 10 a 40 gramas de isca granulada por olheiro, dependendo do diâmetro do olheiro encontrado, conforme preconizado por Anjos (2009). No caso de vizinhança, foram combatidos os sauveiros externos encontrados numa faixa de vegetação natural com largura de 30 metros, partindo da borda da plantação de eucaliptos.

Cerca de seis meses após a realização do combate, foram sorteados talhões de eucalipto, utilizando-se mapas dos projetos, na base de um talhão para cada $400 \mathrm{ha}$, os quais foram examinados quanto à presença de sauveiros externos e ativos. A localização dos focos de atividade de formigas saúvas foi obtida percorrendose os aceiros externos de cada talhão sorteado para encontrar trilhas de forrageamento dos sauveiros externos, associados aos eucaliptos atacados.

A primeira característica estudada foi a distância do sauveiro externo. Para tal, seguiu-se a trilha de forrageamento das formigas até encontrar o correspondente sauveiro; de volta, mediram-se a distância entre o monte de terra solta daquele sauveiro e a margem do plantio. Para verificar uma possível associação entre o tamanho de área de terra solta e a distância de forrageamento das saúvas, foi realizada uma análise de correlação simples; posteriormente buscou-se estabelecer a relação entre as duas variáveis por meio de uma regressão linear simples. Para verificar a normalidade, os dados foram submetidos ao teste de KolmogorovSmirnov e Lilliefors ao nível de significância de $0,05 \%$.

A segunda característica avaliada foi a área de cada sauveiro externo, para a qual se mediram o maior comprimento e a maior largura do monte de terra solta, conforme preconizado por Mariconi e Paiva (1960). Adicionalmente, coletaram-se soldados cujas espécies foram determinadas nos Laboratórios da Universidade Federal de Viçosa, sob a orientação da Dra. Cidália Gabriela Marinho, e depositados no Museu Regional de Entomologia (UFVB).

\section{RESULTADOS E DISCUSSÃO}

Nos 21 talhões examinados, foram encontrados nove sauveiros externos em atividade. Em nenhum dos talhões havia mais do que um sauveiro externo ativo, resultando em $42,86 \%$ de ocorrência desse tipo de sauveiro em tais operações de avaliação. Isso é semelhante ao encontrado por Lima e Anjos (1997), os quais constataram tal ocorrência em $37,31 \%$ dos casos de monitoramentos de formigas cortadeiras. As características dos sauveiros externos encontrados estão disponibilizadas na Tabela 1.

TABELA 1: Características de sauveiros externos remanescentes em plantios de eucaliptos. Fevereiro, 2008.

TABLE 1: Characterization of leaf cutting ant mounds outside eucalypt plantations. February, 2008

\begin{tabular}{lccl}
\hline $\begin{array}{c}\text { Localidade e espécie de } \\
\text { saúva }\end{array}$ & $\begin{array}{c}\text { Distância do sauveiro } \\
(\mathrm{m})\end{array}$ & $\begin{array}{c}\text { Área de terra solta } \\
\left(\mathrm{m}^{2}\right)\end{array}$ & $\begin{array}{c}\text { Espécie de eucalipto } \\
\text { atacado }\end{array}$ \\
\hline Fazenda 1 - João Pinheiro & 14 & & \\
\hline Atta laevigata & 16 & 24 & Eucalyptus urophylla \\
Atta sexdens rubropilosa & 31 & 85 & Eucalyptus camaldulensis \\
Atta sexdens rubropilosa & 51 & 60 & Eucalyptus urophylla \\
Atta sexdens rubropilosa & 70 & 60 & Eucalyptus urophylla \\
Atta sexdens rubropilosa & 127 & 112 & Eucalyptus urophylla \\
Atta sexdens rubropilosa & 45 & 109 & Eucalyptus urophylla \\
\hline Fazenda 1- Buritizeiro & 48 & 84 & Eucalyptus urophylla \\
\hline Atta laevigata & 4 & 30 & Eucalyptus camaldulensis \\
\hline Atta laevigata & & &
\end{tabular}


Em João Pinheiro, MG, a maioria dos sauveiros externos encontrados pertencia à espécie Atta sexdens rubropilosa; apenas um deles era da espécie Atta laevigata. Essas duas espécies de saúvas já haviam sido registradas como ocorrentes em eucaliptais desse município (ZANUNCIO et al., 1997; GRANDEZA et al., 1999; ZANETTI et al., 2000). Em Buritizeiro, as espécies de formigas cortadeiras encontradas foram as mesmas do município de João Pinheiro. Entretanto, esta é a primeira vez que se relata a presença de Atta laevigata e de Atta sexdens rubropilosa nesse Município. Como informação adicional, relata-se também, pela primeira vez, a ocorrência de formigas de uma espécie do gênero Trachymyrmex, em Buritizeiro. As formigas desse gênero já foram mencionadas por Pacheco e Berti Filho (1987), danificando gemas de brotações em touças de eucaliptos, numa infestação média de 32 colônias por hectare. Acrescenta-se, ainda, que foram encontradas formigas cortadeiras de uma espécie do gênero Acromyrmex, nos plantios, dos dois municípios vistoriados, e que ainda não havia sido registrada para tais municípios de Minas Gerais.

A distância média de localização dos sauveiros externos em relação à margem do talhão do eucaliptal atacado, em João Pinheiro, foi de 51,5 $\pm 17,44 \mathrm{~m}$, portanto, além dos $30 \mathrm{~m}$ de largura da faixa de segurança recomendada e usada durante o combate realizado nesses eucaliptais e um pouco além da largura mínima recomendada por Lima e Anjos (1997). Constatou-se que esses sauveiros externos, plenamente ativos após seis meses do combate, estavam localizados a uma distância de até $127 \mathrm{~m}$ da margem do plantio; dois deles, entretanto, estavam a uma distância inferior à largura da faixa de segurança, podendo-se inferir que houve problemas na distribuição do formicida ou no treinamento da equipe de combate. $\mathrm{Na}$ primeira fazenda localizada em Buritizeiro, foram encontrados apenas dois sauveiros ativos e da espécie Atta laevigata, localizados a uma distância média de 46,5 $\pm 1,50 \mathrm{~m}$. Nesse caso, pode-se aceitálos como em situação normal porque estavam fora da faixa de segurança adotada no combate. $\mathrm{Na}$ segunda fazenda, foi encontrado somente um sauveiro externo e ativo, pertencente à espécie Atta sexdens rubropilosa, o qual estava localizado a uma distância de quatro metros do plantio, denunciando uma falha no combate, uma vez que a atenção máxima deveria ser dada à faixa com até $10 \mathrm{~m}$ de largura, pois é nela que se concentram 50\% dos casos de sauveiros externos, antes do combate, conforme constataram Lima e Anjos (1997). A distância média entre todos os sauveiros externos encontrados foi de 33,94 $\pm 8,68 \mathrm{~m}$, portanto um pouco acima da largura recomendada como faixa de segurança, sugerindo a necessidade de ampliar essa largura para os $50 \mathrm{~m}$ preconizados por Lima e Anjos (1997). Considerando que no total das três fazendas vistoriadas foram encontrados apenas três sauveiros externos localizados a mais de $50 \mathrm{~m}$ da margem do plantio e considerando que isto significa $12 \%$ dos sauveiros externos, conforme Lima e Anjos (1997) infere-se que poderia haver 22 sauveiros externos dentro da faixa de $30 \mathrm{~m}$ preconizada, antes do combate realizado há seis meses. Como foram encontrados apenas três sauveiros externos ativos nessa faixa, conclui-se que o restante foi eliminado pela operação de combate, significando uma eficiência de $86,36 \%$ de controle. Nesse tipo de operação é desejável alcançar uma eficiência superior a $95 \%$, o que significa a possibilidade de melhorar o treinamento da equipe e de aperfeiçoar as técnicas de combate às formigas cortadeiras usadas naquela localidade.

Houve uma correlaçãopositiva esignificativa $(\mathrm{r}=0,83 ; \mathrm{n}=6, \mathrm{P} \leq 0,05)$ entre a área de terra solta do sauveiro e a sua distância de forrageamento apenas para Atta sexdens rubropilosa (Figura 1). Dessa forma, sugere-se que sauveiros dessa espécie e com maior área de terra solta, em função da maior quantidade de indivíduos e da maior capacidade de forrageamento, podem forragear a uma distância maior do que a de sauveiros pequenos. Grandeza et al. (1999) constataram que sauveiros com elevadas áreas de terra solta indica que eles são adultos, ou seja, têm mais de três anos de idade. A existência de formigueiros adultos significa a possibilidade permanente de proliferação dessa praga, conforme relataram Della Lucia e Bento (1993), uma vez que são capazes de produzirem, anualmente, grande quantidade de indivíduos reprodutores (MARICONI, 1970).

A área média de terra solta nos sauveiros externos encontrados em João Pinheiro foi de $61 \pm$ $13,96 \mathrm{~m}^{2}$. Os dois sauveiros externos encontrados a uma distância inferior aos $30 \mathrm{~m}$ apresentavam área média de terra solta igual a $24,5 \mathrm{~m}^{2}$, podendose inferir com base nas estimativas de crescimento de ninhos preconizados por Grandeza et al. (1999) que esses formigueiros apresentavam mais do que dois anos de idade e que, portanto, deveriam ter sido combatidos nas operações de combates anteriores.

Ci. Fl., v. 21, n. 2, abr.-jun., 2011 


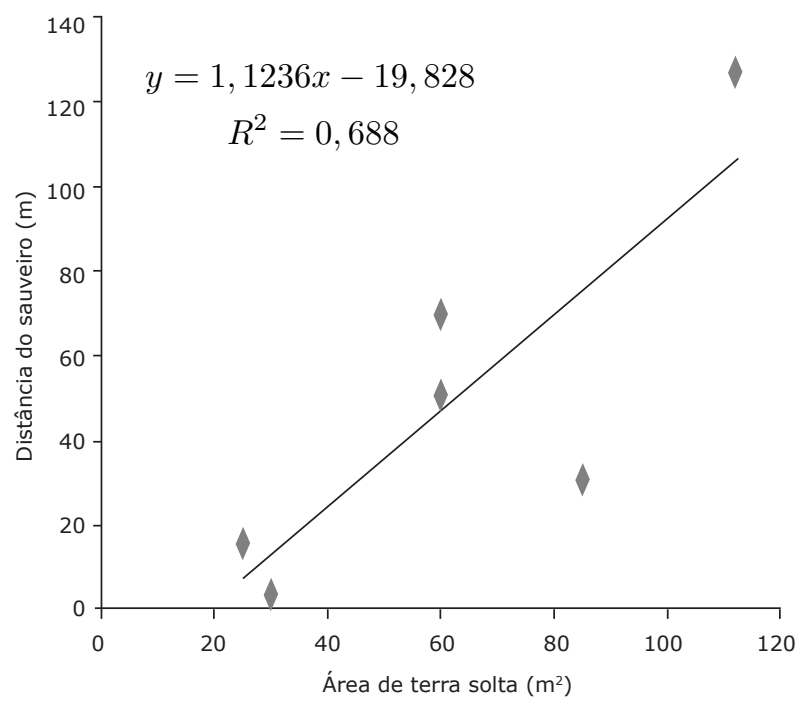

FIGURA 1: Correlação entre o tamanho do sauveiro e a distância de forrageamento em plantios de eucaliptos. Fevereiro, 2008.

FIGURE 1: Correlation between nest size and foraging distance for Atta sexdens rubropilosa leaf cutting ant. February, 2008.

Em Buritizeiro, os dois sauveiros da espécie Atta laevigata, encontrados na primeira fazenda, apresentaram área média de terra solta de 96,5 $\pm 12,5 \mathrm{~m}^{2}$. Na segunda fazenda, o único sauveiro externo encontrado apresentava uma área de terra solta estimada em $30 \mathrm{~m}^{2}$. Considerando todas as avaliações feitas nas três fazendas, os sauveiros externos apresentaram média geral de terra solta de $62,5 \pm 11,1 \mathrm{~m}^{2}$ e a maior área de terra solta encontrada foi de $109 \mathrm{~m}^{2}$, o que não representam nenhuma anormalidade já que, segundo Della Lucia et al. (2000), é comum encontrar sauveiros com área de terra solta superior a $200 \mathrm{~m}^{2}$.

EmJoãoPinheiro,MG, amaioriadossauveiros externos estava atacando Eucalyptus urophylla, e apenas um formigueiro de Atta sexdens rubropilosa estava atacando Eucalyptus camaldulensis. Grandeza et al. (1999) já haviam relatado a presença de Atta laevigata e de Atta sexdens rubropilosa em plantios comercias de Eucalyptus camaldulensis nesse Município. Entretanto, em Eucalyptus urophylla, essas duas espécies não haviam sido relatadas ainda e podem ser consideradas como uma nova ocorrência em João Pinheiro, MG. No município de Buritizeiro, não havia registro científico de Atta laevigata em nenhuma espécie florestal, sendo esta a primeira ocorrência da espécie em Eucalyptus urophylla.

\section{CONCLUSÕES}

O estudo de características inerentes aos sauveiros externos, que sobreviveram após uma operação de combate, é de grande utilidade no aperfeiçoamento da equipe de combate, das técnicas adotadas e das estratégias necessárias para se conseguir diminuir os estragos causados por formigas cortadeiras numa cultura de eucaliptos. Dado o exposto, pode-se concluir que a faixa de combate utilizada deve ser de $50 \mathrm{~m}$ de largura.

\section{AGRADECIMENTOS}

À empresa COMPANHIA FERROLIGAS MINAS GERAIS - MINASLIGAS, pelo apoio e autorização para coleta de dados em suas propriedades. Agradecimentos são devidos também à Dra. Cidália Gabriela Marinho pelas identificações das espécies de formigas cortadeiras e ao estudante Paulo Roberto Magistrali pela ajuda na coleta dos dados.

\section{REFERÊNCIAS BIBLIOGRÁFICAS}

ANJOS, N. Sistema Arrastão para combate a formigas cortadeiras. In: SIMPÓSIO DE MIRMECOLOGIA, 21., 2009, Ouro Preto - MG. Anais... Ouro Preto, 2009. p. 101-101.

ANJOS, N.; DELlA LUCIA, T. M. C.; MAYNUNES, A. J. Guia prático sobre formigas cortadeiras em reflorestamentos. Ponte Nova: Graff Cor, 1998. 100 p.

BERGMANN, E. C. et al. Contribuição ao estudo da fauna mirmecológica associada a seringueira (Hevea brasiliensis). In: ENCONTRO DE MIRMECOLOGIA, 10., 1995, Piracicaba. Anais... Piracicaba, 1995. p. 4-4.

DELLA LUCIA, T. M. C.; ANJOS, N.; ZANUNCIO, J. C.; Controle de formigas cortadeiras. Viçosa, CTP, 2000, 52 p.

DELLA LUCIA, T. M. C.; BENTO, J. M. S. Vôo nupcial ou revoada. In: DELLA LUCIA, T. M. C. (Ed). As formigas cortadeiras. Viçosa, 1993, 262 p.

GARCIA, I. P.; FORTI, L. C. Atividade forrageira e construção de trilhas da saúva Atta sexdens rubropilosa Forel (Hymenoptera: Formicidae) em Eucalyptus grandis e mata secundária. In: CONGRESSO BRASILEIRO DE ENTOMOLOGIA, 15., 1995, Caxambu. Anais... Caxambu, 1995. p. 553-553. 
GARCIA, I. P.; FORTI, L. C.; ENGEL, V. L. Seletividade de plantas por Atta sexdens L., 1758 (Hym., Formicidae) em um fragmento de floresta estacional semidecidual: interferência de parâmetros fitossociológicos. In: ENCONTRO DE MIRMECOLOGIA, 15., 2001, Londrina. Anais... Londrina, 2001. p. 97-100.

GRANDEZA, L. A. O.; MORAIS, J. C.; ZANETTI, R. Estimativa do crescimento externo de ninhos de Atta sexdens rubropilosa Forel e Atta laevigata (F. Smith) (Hymenoptera: Formicidae) em áreas de reflorestamento com eucalipto. Anais Sociedade de Entomologia Brasil, Londrina, v. 28, n. 1, p. 59-64, 1999.

LIMA, R. C. A.; ANJOS, N. Combate a formigas cortadeiras em vizinhança de eucaliptais: faixa de segurança. In: INTERNATIONAL PEST ANT SYMPOSIUM, 6.; e ENCONTRO DE MIRMECOLOGIA, 13., 1997, Ilhéus. Anais... Ilhéus, 1997. p. 181-181.

MARICONI, F. A. M,; PAIVA, C. Notas sobre a saúva e o sauveiro. O Biológico, São Paulo, v. 26, n. 6 , p. $97-108,1960$.

MARICONI, F. A. M. et al. Nova contribuição para o conhecimento das saúvas de Piracicaba (Atta spp.) (Hym. Formicidae). Revista de Agricultura, Piracicaba, v. 38, n. 2, p. 85-93, 1970.

MARSARO JUNIOR, A. L. Formigas cortadeiras em plantios de Acacia mangium Willd. (Fabaceae - Mimosoideae): Identificação e Controle. EMBRAPA. Comunicado Técnico. Boa Vista, p. 4. 2005.

MOREIRA, R. T. S. Teste de preferência de forrageamento de Atta sexdens rubropilosa, Forel, 1908, por três espécies de eucalipto no campo.
2006. 28 f. Monografia (Curso de Engenharia Florestal)-Universidade Federal Rural do Rio de Janeiro, Rio de Janeiro, 2006.

OLIVEIRA, M. A. Identificação de formigas cortadeiras e efeito do desfolhamento simulado em plantios de Eucalyptus grandis. 1996. 61 f. Dissertação (Mestrado em Entomologia)-Universidade Federal de Viçosa, Viçosa, 1996.

PACHECO. P.; BERTI FILHO. E. Formigas cortadeiras e seu controle. IPEF, 1987, p. 152.

SCHOEREDER, J. H. Aspectos ecológicos da saúva no cerrado. São Paulo: USP, 1985. 112 f. Dissertação (Mestrado em Ciências) - Universidade de São Paulo, 1985.

SILVA, E. J. Identificação de formigas cortadeiras e efeito do desfolhamento simulado em plantios de seringueira (Hevea brasiliensis Mull Arg.). 2001. 43 f. Dissertação (Mestrado em Entomologia)Universidade Federal de Viçosa.

VASCONCELOS, H. L. Foraging activity of an Amazonian leaf-cutting ant: responses to changes in the availability of woody plants and to previous plant damage. Oecologia, Heidelberg, v. 112, p. 370-378, 1997.

ZANETTI, R. et al. Influência da espécie cultivada e da vegetação nativa circundante na densidade de sauveiros em eucaliptais. Pesquisa Agropecuária Brasileira, Brasília, v. 35, n. 1, p. 1911-1918, jan. 2000.

ZANUNCIO, J. C. et al. Uso da isca granulada com Sulfluramida 0,3 \%, no Controle de Atta sexdens rubropilosa Forel, 1908 (Hymenoptera: Formicidae). Cerne, Lavras, v. 3, n.1, p. 161-169, 1997. 